\title{
A full characterisation of the GELINA neutron flux distributions and resolution functions with the Monte Carlo code MCNP5
}

\author{
D. Ene ${ }^{1}$, C. Borcea ${ }^{1,2}$, M. Flaska ${ }^{3}$, S. Kopecky ${ }^{1}$, A. Negret ${ }^{1, a}$, W. Mondelaers ${ }^{1}$, and A.J.M. Plompen ${ }^{1, b}$ \\ ${ }^{1}$ European Commission, Joint Research Centre, Institute for Reference Materials and Measurements, 2440 Geel, Belgium \\ 2 National Institute for Physics and Nuclear Engineering "Horia Hulubei", P.O. Box MG-6, Bucharest, Romania \\ 3 Oak Ridge National Laboratory, P.O. Box 2008, Oak Ridge Tennessee, USA
}

\begin{abstract}
A numerical analysis of the GELINA target-moderator assembly has been conducted with the MCNP5 code to completely characterise the facility's neutron and gamma flux distributions as well as the neutron resolution functions. The aim of the work is to provide reference flux distributions for all flight paths for the benefit of external users, improved resolution functions for resonance analysis, and a general purpose calculational tool that may be used as a starting point for the optimisation of experimental arrangements. This work is based on an earlier study in which the suitability of the use of the MCNP code was demonstrated for the purposes discussed here. This detailed neutronic study with the MCNP5 code was performed for all flight paths of the facility. The following parameters characterising the performance of a neutron time-of-flight facility were investigated for the fast and for the moderated flux configurations:

- the energy distribution of the time-integrated neutron flux,

- the resolution function in dependence of the neutron energy and flight path angle,

- the gamma background at the detector position,

- the time distribution of neutrons and photons at the detector location.

The quality of the facility was also summarised by means of the energy-dependent figure of merit. Results of these calculations are presented and discussed. A better understanding of the correlations between the energy and the time of flight as well as of the origin of the components contributing to the spectra has been achieved by analysing time of flight spectra of both neutrons and photons with respect to the time at which the electron burst hits the target. Based on this work, the accuracy of the determination of the nuclear resonance parameters from time-of-flight measurements was further improved by implementing the detailed description of the resolution functions in the analysis code REFIT. Implementation and testing in the REFIT code of the obtained resolution functions is discussed.
\end{abstract}

\section{Introduction}

The pulsed white neutron source GELINA is used for high resolution and high accuracy neutron cross section measurements with the time-of-flight technique. Such cross sections are of great importance for the estimation of reaction rates and neutron flux distributions in various applications related to nuclear power reactors and new developments such as the Generation-IV and accelerator driven systems. Reducing uncertainties of nuclear data may lead to significant improvements in the safety margins and the reliability of calculated performances for new reactor concepts. A number of recent examples of measurements at the GELINA facility may be found elsewhere, in these proceedings [1-10] and through references in these works.

The present effort to obtain a complete albeit generic characterisation of the facility by Monte Carlo simulations was motivated as follows. New demands for improved nuclear data aim for higher precision of the important reactions on major actinides, for increased emphasis on the fast neutron energy range and for studies of innovative materials, i.e. fuels, fuel matrices, coolants and structural materials. Such new requirements, call for improved characterisation of measurement

\footnotetext{
${ }^{a}$ On leave of absence from IFIN-HH, Bucharest, Romania

${ }^{b}$ Presenting author, e-mail: Arjan.Plompen@ec.europa.eu.
}

conditions and for the design of new experiments. For GELINA one further development is the increased involvement of external parties performing measurements at the facility. In order to provide guidance to those proposing new experiments it is of importance that the basic characteristics of the neutron source are well understood. Therefore a complete survey was undertaken using the MCNP5 Monte Carlo transport code for the neutron and gamma flux and the neutron and gamma energy and angle distribution. Furthermore, a complete determination of neutron resolution functions was accomplished and implemented in the REFIT resonance analysis code, thus enhancing the confidence in resonance shape analysis. Resonance shape analysis is of interest in parametrising the results of cross section measurements in the resolved resonance region and in dealing with the effects of instrumental and Doppler broadening of resonances. Finally, the present characterisation effort has left us with a simulation tool that is a starting point for the estimation of count rate, background, collimation and shielding of importance to the experiments planned and being carried out.

A comprehensive account of this characterisation effort was submitted for publication [11]. In view of the extent of the work only a brief summary can be presented here. The present work is based on an earlier study by M. Flaska et al., that established the validity and method of operation for the 
use of MCNP for the purpose of modeling the GELINA source [12]. M. Flaska et al. also used the developed methodology to establish the potential for improved fast neutron measurements at GELINA [13]. Further work along these lines but for the moderated spectrum and for a compromise between the moderated and the direct spectrum was recently completed and will be described elsewhere.

\section{The GELINA facility}

The Geel electron linear accelerator (GELINA) produces intense pulses of neutrons of $1 \mathrm{~ns}$ duration. A primary electron pulse train of $10 \mathrm{~ns}$ consisting of 30 pulses is accelerated to energies from 140 for the first to $70 \mathrm{MeV}$ for the last pulse. The pulse train is compressed to one single burst of $1 \mathrm{~ns}$ with a large dipole magnet before striking a rotating disk-shaped ${ }^{238} \mathrm{U}$ target [14]. On the uranium, bremsstrahlung is generated and neutrons follow from ${ }^{238} \mathrm{U}(\gamma, n),{ }^{238} \mathrm{U}(\gamma, 2 n)$ and ${ }^{238} \mathrm{U}(\gamma, F)$ (20\%) reactions [12]. The neutron spectrum emerging from the uranium is a composite evaporation and fission neutron spectrum with a useful range from several $\mathrm{keV}$ to a few tens of $\mathrm{MeV}$. To extend the useful range to $10 \mathrm{meV}$, i.e. just below thermal, two beryllium cans with water are placed above and below the target to moderate the neutrons.

Eighteen flight paths view the neutron producing target of which seven are used routinely. Flight paths are evacuated $\mathrm{Al}$ tubes with a diameter of $50 \mathrm{~cm}$. Measurement equipment may be placed at distances ranging from 8 to $400 \mathrm{~m}$. Flight paths either shadow the uranium target to view only the moderator in order to obtain neutrons from $10 \mathrm{meV}$ to $1 \mathrm{MeV}$ or they shadow the moderators when the measurements concern the range from $100 \mathrm{keV}$ to $20 \mathrm{MeV}$. The first shadowing configuration is termed the Moderated Flux Configuration (MFC), the second the Direct Flux Configuration (DFC). At present, state-of-the-art measurement equipment is installed for measurements of the total, capture, fission, inelastic, (n,2n) and (n,chp) cross sections.

\section{Monte Carlo model of the facility}

The Monte Carlo code MCNP5 is used for the simulations. Coupled electron-photon-neutron transport is used with the accelerated electrons of GELINA as the source particles. The photo-neutron libraries developed through a Co-ordinated Research Project of the IAEA are used for the $(\gamma, x n)$ and $(\gamma, F)$ reactions taking place in the target. The Monte Carlo model of the facility consists of two parts. The first part is for the neutron producing target while the second part is a generic model for the eighteen flight paths. The model for the target was developed prior to the present effort by M. Flaska et al. [12] for version 4.3C of the MCNP code. The composite construction sketch and the actual model are shown in figures 1 and 2 . The model flight paths are fully evacuated. Idealised shadow bars and two idealised collimators are used with a measurement point at $32 \mathrm{~m}$ from the center of the neutron production distribution. At the entrance the collimator has a diameter of $16.8 \mathrm{~cm}$ while $2 \mathrm{~m}$ in front of the measurement it is $12 \mathrm{~cm}$ diameter for the MFC and $3 \mathrm{~cm}$ for the DFC.

In ref. [12] an absolute comparison was made of calculated and measured fluxes for the direct and the moderated flux

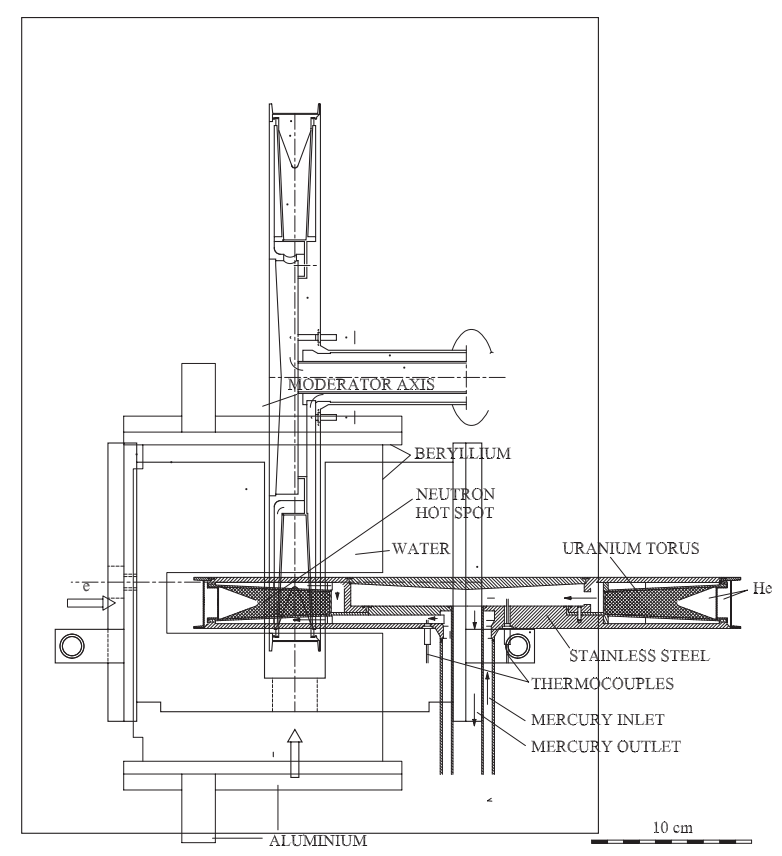

Fig. 1. Composite construction sketch of the GELINA rotary target.

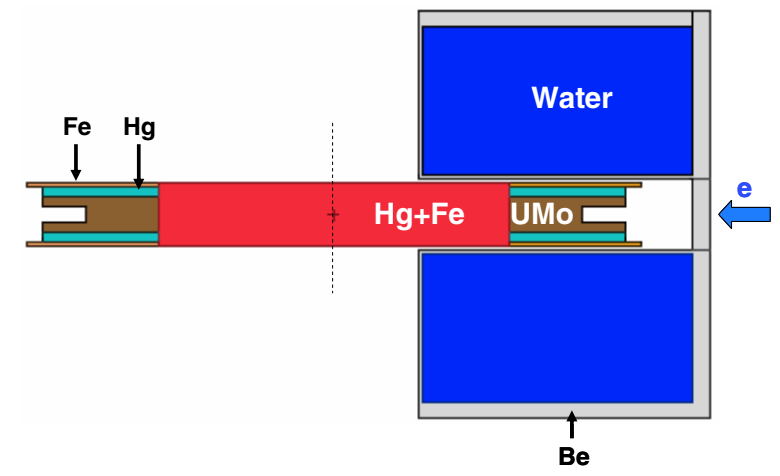

Fig. 2. MCNP model of the GELINA rotary target.

configuration of GELINA showing very satisfactory agreement (20\%). Also in that work, a (tallyx) subroutine was made to score the so-called delay distance $d$ given by

$$
d=v t-L
$$

where $v$ is the velocity, $t$ the time and $L$ the distance to the nominal source location of the neutron at the tally ${ }^{1}$ point. The delay distance is a convenient quantity for the study of the neutron resolution functions - RF(E,d). An RF is the probability distribution for the arrival time at the measurement (tally) point of neutrons with energy $E$. RFs as function of time are strongly varying functions of energy, while RFs as function of delay distance are very modest functions of energy.

\section{Results}

A brief overview of the results of the simulations is presented along with an illustration of the use of the new resolution functions.

\footnotetext{
${ }^{1}$ A tally is the MCNP designation for a "measurement" in the Monte Carlo simulation.
} 


\subsection{Neutron and gamma flux}

Figures 3 and 4 show the total neutron and gamma flux for the direct and moderated flux configurations in function of the flight path angle. A strong angle dependence is observed for the DFC neutron flux and even more dramatically so for the gamma flux. This is the consequence of the self-shielding of the rotary target. Neutrons are produced in a volume of a few cubic centimeters at about $10 \mathrm{~mm}$ from the surface that is struck by the electron beam. Flight paths using the direct spectrum view this hot spot in the plane of the rotary target, whence the strong self-shielding.

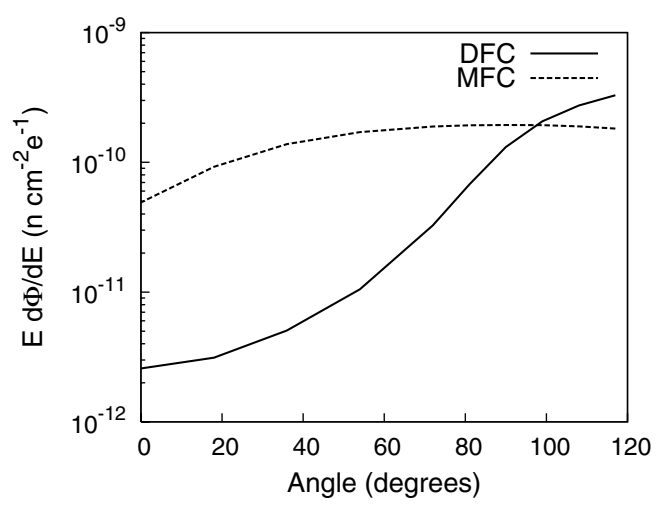

Fig. 3. Total neutron flux versus flight path angle.

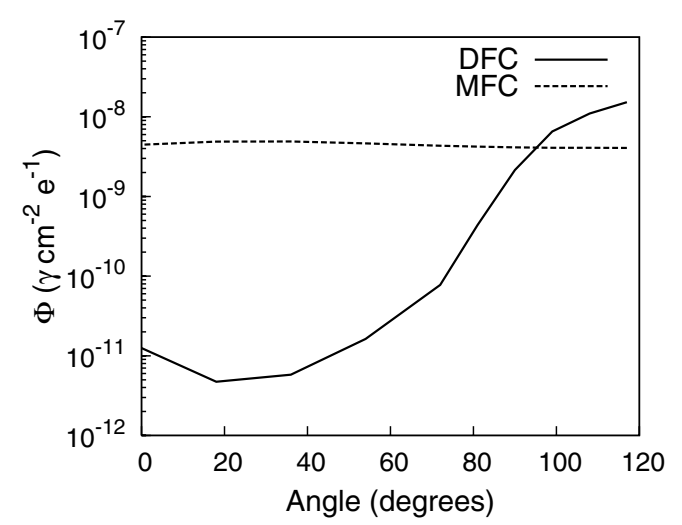

Fig. 4. Total gamma flux versus flight path angle.

For the moderated flux configuration the angle dependence is considerably less. For neutrons an angle dependence from 0 to about 50 degrees with respect to the electron beam is apparent which results from self-shielding of the moderator due to its shape and orientation. For gammas practically no angle effect is evident. Gammas for the MFC originate from pair production and Compton scattering of gamma-rays emerging from the uranium and incident on the water moderator and the Be can. Due to the low $\mathrm{Z}$ of both water and Be there is practically no self-shielding of the gammas.

\subsection{Neutron flux distribution}

Neutron flux distributions were obtained for all flight path angles and for both moderated and direct flux configurations.

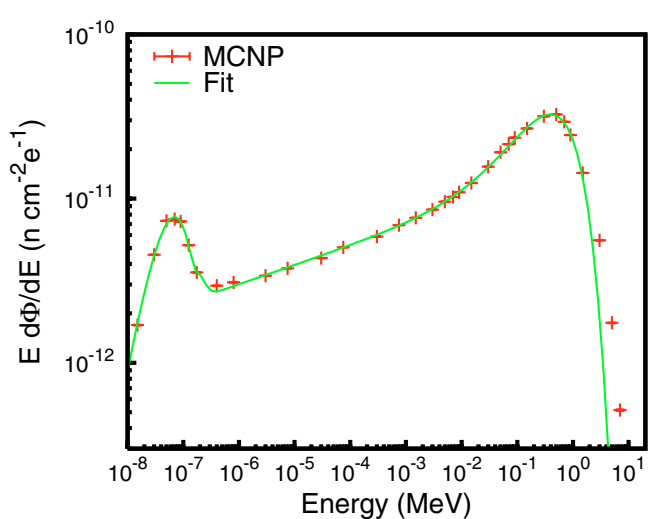

Fig. 5. Energy distribution for neutrons in the moderated flux configurations at 90 degrees with respect to the electron beam.

A typical example is shown in figure 5 for the MFC. For the MFC the characteristic features are the Maxwellian thermal component below $0.5 \mathrm{eV}$, the power law in the slowing down region from $0.5 \mathrm{eV}$ to $20 \mathrm{keV}$, and the source region from $20 \mathrm{keV}$ to $10 \mathrm{MeV}$. To illustrate these contributions and to arrive at a convenient representation for count rate estimates, a fit was made based on the following function which was inspired by an expression proposed by Carpenter for the energy region below $20 \mathrm{keV}$ [16]. The function provides a good description for the MFC from $10 \mathrm{meV}$ to $1 \mathrm{MeV}$ and for all angles, however with angle dependent parameters.

$$
\Phi(E)=I_{t} \frac{E^{2}}{T^{2}} e^{-\frac{E}{T}}+I_{e} f_{c}(E)\left(\frac{E}{E_{r}}\right)^{a}\left(1+r\left(\frac{E}{E_{r}}\right)^{b}\right) e^{-\frac{E}{\theta}}
$$

Here, $I_{t}$ is the intensity of the Maxwellian component, $T$ the effective moderator temperature, $I_{e}$ the intensity of the epithermal component, $f_{c}$ a low energy cut-off function for the epithermal component, $E_{r}=1 \mathrm{eV}, a$ is the exponent of the epithermal power law, $r$ is the ratio of the intensity of the source component to that of the epithermal component, $b$ is the corresponding power and $\Theta$ the corresponding temperature. $I_{e} / I_{t}$ varies from 0.27 at $0^{\circ}$ to 0.21 at $90^{\circ}, T=34.8 \mathrm{meV}$ and $a=0.117$ both nearly independent angle, $b$ varies from 1.1 at $0^{\circ}$ to 0.68 at $90^{\circ}, \Theta$ from 0.68 to $0.61 \mathrm{MeV}$. The angle variation of $r$ is the most dramatic ranging from nearly zero at $0^{\circ}$ to $5.7 \cdot 10^{-4}$ at $90^{\circ} . I_{t}\left(90^{\circ}\right)=1.4 \cdot 10^{-11} \mathrm{n} / \mathrm{cm}^{2} / \mathrm{e}$.

\subsection{Neutron resolution functions}

Figures 6 and 7 show the neutron resolution functions in function of the delay distance and versus energy for the flight path at 90 degrees with respect to the electron beam. Results are shown for both the moderated and the direct flux configuration. Similar results were obtained for all other flight paths. It is clearly seen that these functions are relatively modest functions of energy, as mentioned earlier. However, when inspected carefully the energy dependence can not be neglected in any energy domain with the exception of the region from $1 \mathrm{eV}$ to about $2 \mathrm{keV}$. The merits of the present work compared to earlier work of Coceva and Magnani [17] for our facility lies in the finer binning with regard to delay distance 


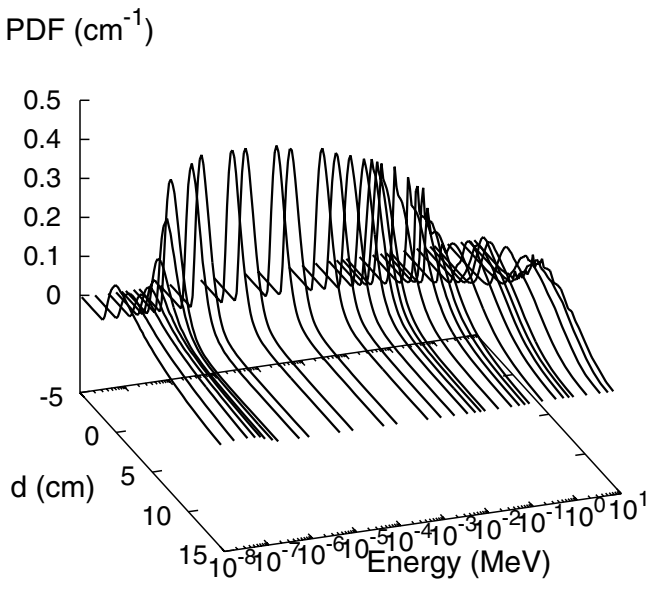

Fig. 6. Neutron resolution functions for the moderated flux configuration at 90 degrees with respect to the electron beam.

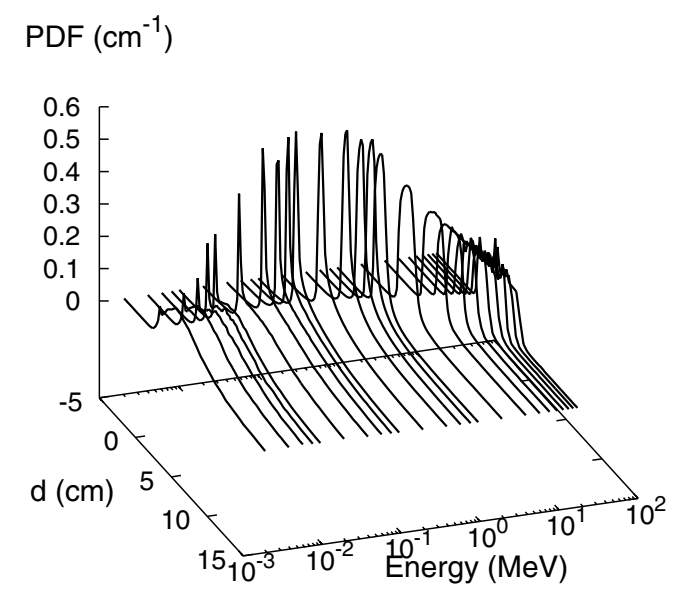

Fig. 7. Neutron resolution functions for the direct flux configuration at 90 degrees with respect to the electron beam.

and energy and the use of a well validated and flexible Monte Carlo code for which routine variance reduction schemes can be used to avoid approximations in the simulation.

\subsection{Resolution functions versus experiment}

The calculated resolution functions were implemented by a table-interpolation method in the resonance shape analysis code REFIT [18]. To check these resolution functions resonance shape analysis was carried out for recently obtained transmission factors and capture yields. Resonances in ${ }^{103} \mathrm{Rh}$ and ${ }^{56} \mathrm{Fe}$ were selected for which the natural line widths are negligible and the Doppler broadening is small. Good agreement was obtained [11] and an example of this is shown in Figure 8 for the $34 \mathrm{keV}$ resonance of ${ }^{56} \mathrm{Fe}$. The dominant part of the width and in particular, the asymmetry of the line shape are due to the resolution function. Furthermore a systematic difference in extracted neutron widths was observed moving from the REFIT intrinsic prescription of the RF for the GELINA facility to the results for the RF of the present

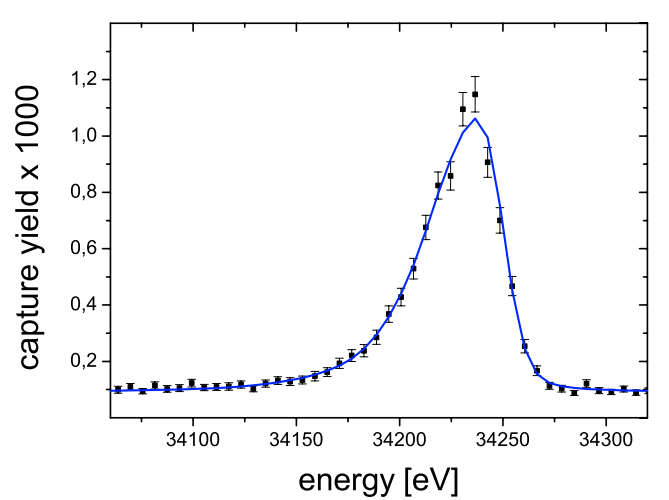

Fig. 8. Capture yield for the $34 \mathrm{keV}$ resonance in ${ }^{56} \mathrm{Fe}$ measured at $60 \mathrm{~m}$ using C6D6 detectors. The apparent full width at half maximum (fwhm) is $40 \mathrm{eV}$. The corresponding fwhm of the resolution function is $33 \mathrm{eV}$. The fwhm for the Doppler effect using the free gas model is $13 \mathrm{eV}$ while the natural width is less than $2 \mathrm{eV}$.

work. The difference, although at the level of a few percent, is of interest in case high precision results are required for a particular nucleus.

\section{Outlook}

A comprehensive understanding of the gamma energy distributions and their origins as deduced from time-of-flight simulations could not be elaborated on here. See Ref. [11] for details. To facilitate use of the present results parametrisations are required to provide an easy method for flux distribution calculations and for a more efficient implementation of resolution functions for resonance shape analysis. Further studies for an improved GELINA neutron producing targets are underway using the present methodology and in connection with earlier work [13].

\section{References}

1. L.C. Mihailescu et al. Nucl. Phys. A 786, 1 (2007).

2. S. Kopecky, A. Brusegan, Nucl. Phys. A 773, 173 (2006).

3. A. Borella et al. Nucl. Sci. Eng. 152, 1 (2006).

4. C. Borcea et al. (these proceedings).

5. A. Borella et al. (these proceedings).

6. S. Kopecky et al. (these proceedings).

7. L.C. Mihailescu et al. (these proceedings).

8. A. Negret et al. (these proceedings).

9. P. Schillebeeckx (these proceedings).

10. C. Wagemans et al. (these proceedings).

11. D. Ene et al. (submitted for publication).

12. M. Flaska et al., Nucl. Instrum. Meth. A 531, 394 (2004).

13. M. Flaska et al., Nucl. Instrum. Meth. A 555, 329 (2005).

14. D. Tronc et al., Nucl. Instrum. Meth. A 228, 217 (1985).

15. IAEA-TECDOC-1178, IAEA, Vienna, Austria (2000).

16. J.M. Carpenter, www.neutron.anl.gov/NeutronProduction.pdf, (2004).

17. C. Coceva, A. Magnani, Report GE/R/ND/06/96, EC-JRCIRMM, unpublished (1996).

18. M. Moxon, Report AEA-inTec-0470, UKAEA, Harwell, UK, unpublished (1991). 